\title{
INTERFACE DETECTION OF OIL-WATER STRATIFIED FLOW
}

\author{
KSHANTHI PERERA, MADURANGA AMARATUNGA \& RUNE W. TIME \\ Department of Energy and Petroleum Engineering, University of Stavanger, Norway
}

\begin{abstract}
Interfacial shape of stratified flow of two liquids in pipes may take a planar or curved shape depending on the physical properties of the fluids, wall-fluid wettability, the geometrical dimensions and the fluids hold-up. It is traditionally accepted that the interfacial curvature is present in capillary and small-scale systems where the surface tension effect is significant against gravity effects. However, it is possible that interfacial curvature is present in liquid-liquid systems with small density differences or in reduced gravity systems due to dominating surface phenomena. Two phase flow of oil (density $=788 \mathrm{~kg} / \mathrm{m}^{3}$, viscosity $=1.6 \mathrm{mPa} . \mathrm{s}$ ) and water (density $=997 \mathrm{~kg} / \mathrm{m}^{3}$, viscosity $=1 \mathrm{mPa} . \mathrm{s}$ ) in a horizontal pipe was investigated for stratified flows. The longitudinal view was recorded using high-speed video imaging, while the cross sectional view of the flow was captured via Electrical Capacitance Tomography (ECT). As a third method, the interfacial level at the mid-pipe was calculated by referring to a work reported in literature. In addition, the interfacial level and the curvature in stratified smooth flow (ST), were calculated using CFD simulations as well. The ECT images indicated a blurred interfacial margin where the interface was reconstructed with a considerable thickness. However, the interfacial level at the pipe wall shown by the cross sectional ECT images were comparable with that of the high-speed images and the CFD simulations. Nevertheless, a significant interfacial curvature was encountered in ECT images towards the mid-pipe, which is 4.3 times deeper than the calculated value. CFD results agreed well with the calculated interfacial level using constant curvature arc model. In ECT, the depth of the curvature at the mid pipe seemed to be far more than the reality due to the possible field distortion effects occurring when the electrical flux lines pass through the media of high permittivity contrast (oil-water). Therefore, it was found that ECT can predict the interfacial oil-water level at the walls with acceptable accuracy, while it over-predicts the interfacial curvature present in the mid-pipe region. It is important to note that the ECT electrodes have their highest sensitivity near the wall region.

Keywords: oil-water stratified flow, interfacial curvature, electrical capacitance tomography, highspeed imaging, CFD.
\end{abstract}

\section{INTRODUCTION}

The work by Taitel and Dukler [1] is one of the initial attempts to model two phase stratified flow by applying separate conservation equations for each fluid stream of gas-liquid flow in which they have assumed a smooth flat interface.

According to Brauner et al. [2], when two immiscible liquids flow through a pipeline, the free interface could take a planar or curved shape depending on the physical properties of the fluids, wall-fluid wettability, pipe geometry and the fluids hold-up. They compared the interface of gas-liquid and liquid-liquid stratified flows. Gas-liquid flows are more likely to have a flat interface due to considerable density differences between the fluids and thus dominant gravity forces that overcome the interfacial forces. However, liquid-liquid flows are prone to possess a curved interface especially when the densities of the fluids are matching and interfacial tension overcomes the effect of gravity.

Research done on the area reveal that the consideration of the interfacial shape is crucial for accurate prediction of pressure drop, hold up and the multiphase flow transport phenomena [3], [4]. Brauner et al. [5] predicted the interfacial configuration for two-fluid systems, based on the energy considerations. They claim that, the interface could get a steady, curved shape so that the total static energy of the system reaches a minimum. The steady 
interface curvature is characterized based on density difference of the fluids, surface tension, gravity conditions and the pipe diameter [2] and those can be embodied into a single nondimensionless number called the Eötvös $\left(\epsilon_{\mathrm{v}}\right)$ number (or sometimes the "Bond number") as shown in eqn (1) [3]

$$
\epsilon_{v}=\frac{\left(\rho_{w}-\rho_{o}\right) g D^{2}}{4 \sigma_{w o}} .
$$

Here, $\sigma(\mathrm{N} / \mathrm{m})$ is the interfacial tension, $\rho\left(\mathrm{kg} / \mathrm{m}^{3}\right)$ is the fluid density, $g\left(\mathrm{~ms}^{-2}\right)$ is the gravitational acceleration, $D(\mathrm{~m})$ is the pipe diameter and $w$ and $o$ denotes the water and oil phases. For larger values of Eötvös number (when $\epsilon_{v} \rightarrow \infty$ ), the interface attains a flat shape and when $\epsilon_{\mathrm{v}} \rightarrow 0$, it becomes a constant curvature curve. The interface configuration also depends on additional system parameters such as fluids hold-up and fluid-wall relative wettability (contact angle) [6]. Brauner et al. [2] presented a straightforward extension of the two-fluid model for analysing stratified flow with curved interfaces. Further, flow diagrams were developed using this model, based on the $\epsilon_{\mathrm{v}}$ number, solid-liquid contact angle, and the phase distribution angle for estimating the interfacial curvature. Sunder Raj et al. [4] reformulated the model to estimate the hold-up and validated that with experimental data. Gorelik and Brauner [6] predicted the interface location in stratified flows by obtaining analytical expressions for the interface shape and for the capillary pressure based on system free energy minimization concept. Exact solutions were obtained for defined hold-up, contact angle and the $\epsilon_{\mathrm{v}}$ number. It was shown that the constant curvature model provides a good description of the interfacial shape.

$\mathrm{Ng}$ et al. [7] described the possible interfacial shapes as portions of a family of curves described by a single parameter which is consisted of the Eötvös $\left(\epsilon_{\mathrm{v}}\right)$ number and a reference curvature at the centre of the interface. $\mathrm{Ng}$ et al. [8] continued this work by calculating the velocity fields, shear stresses and flow rates for fully developed, laminar stratified flows. In that work, the interfacial shape was considered according to $\mathrm{Ng}$ et al. [7] and assumed to be free of instabilities. Only the integrals on the fluid-fluid interface were required to determine all the integral and local flow properties of the flow when the BEM (Boundary Element Method) is used.

Rodriguez and Baldani [3] proposed a model based on a constant curvature arc approximation to represent the interfacial shape especially for the liquid-liquid flow, where relatively small $\epsilon_{\mathrm{v}}$ numbers present. The proposed model implicitly accounts for interface shape, turbulence and wavy structure via new closure relations for interfacial and wall shear stresses. Further, the proposed equation for the curvature radius is a function of $\epsilon_{\mathrm{v}}$ number, contact angle and interfacial water height. This model will be further discussed in Section 2.2 , with relevant to the current study.

Electrical capacitance tomography (ECT) is a soft field sensing technique, which can be used to visualize and measure the cross-sectional multiphase flow distribution based on the permittivity differences within the sensor plane. The acquired details are highly dependent on the permittivities of the fluids, image reconstruction algorithm and the size of the pixel grid. The details of the technique used is described in detail by Perera et al. [9].

The focus of this study is to investigate the ability of ECT to reveal the interfacial shape of oil-water stratified flows. High-speed camera image analysis (HIA) and solution of the equation for the curvature radius (based on constant curvature arc model) as proposed by Rodriguez and Baldani [3] will be deployed for comparison. Finally, the interfacial shape predicted by computational fluid dynamics (CFD) will be discussed for stratified smooth (ST) flows. 


\section{MATERIALS AND METHODS}

\subsection{Experimental setup and the test fluids}

The experiments were performed using the multiphase flow rig at University of SouthEastern Norway (USN). The test pipe is $56 \mathrm{~mm}$ ID and $15 \mathrm{~m}$ long stainless steel pipeline. A schematic view of the flow loop can be found in Perera et al. [9]. The test section has a transparent acrylic box filled with water to avoid light reflection, placed approximately $11 \mathrm{~m}$ from the pipe inlet. The oil-water flow was filmed with a high-speed video camera (Photron FASTCAM) via this transparent viewing box. High-speed images were recorded at a rate of 500 fps (frames per second) and a ruler, drawn on the viewing box, was used to achieve the real length-to-pixel conversion. The contact angle for acrylic when in contact with oil-water is considered as $110^{\circ}$ [3]. ECT sensor, which is mounted after the viewing section enabled the cross sectional tomographic flow imaging at a rate of $100 \mathrm{~Hz}$. The details of ECT sensor can be found in Perera et al. [9].

The density and the viscosity of water are $997 \mathrm{~kg} / \mathrm{m}^{3}$ and $1 \mathrm{mPa} . \mathrm{s}$ respectively at temperature of $20^{\circ} \mathrm{C}$. The type of oil is Exxsol D60 with a density of $788 \mathrm{~kg} / \mathrm{m}^{3}$ and a viscosity of $1.6 \mathrm{mPa}$.s at $20^{\circ} \mathrm{C}$. The oil-water interfacial tension is $43 \mathrm{mN} / \mathrm{m}$. The oil-water flow rates were set according to the desired water cut $\left(\lambda_{\mathrm{w}}\right)$ and mixture velocity $\left(\mathrm{U}_{\mathrm{mix}}\right)$. Input water cut $\left(\lambda_{\mathrm{w}}\right)$ is the ratio of water volume flow rate to the total input volumetric flow rate and mixture velocity $U_{\text {mix }}(\mathrm{m} / \mathrm{s})$ is the ratio of total input volumetric fluid flow rate to the cross-sectional area of the pipe. The flow was allowed to stabilize for at least 10 minutes before the measurements.

\subsection{Data treatment and analysis}

Matlab programs were written to extract the image data for detecting the interface from ECT images and high-speed camera images. For high-speed camera images, a sharp interface was assumed and the brightest pixel in each pixel column was detected as the interface. For ECT images the interface was observed as a layer with a considerable thickness, hence threshold data values were set to detect the upper and lower interfacial margins. The oil-water interfacial margin at the pipe wall was obtained via image analysis performed on ECT and high-speed images. The oil-water interfacial margin at the mid-pipe was obtained via ECT image analysis and as a second method, the constant-curvature-arc model presented by Rodriguez and Baldani [3] were used to calculate the interfacial height at the mid-pipe.

Fig. 1 shows the geometrical description of the cross sectional view of oil-water stratified flow. Here, the oil-water interface is assumed as a constant arc of a circle, where, $\emptyset^{*}$ is the interfacial angle formed due to the curved interface and $\mathrm{r}$ is the interface curvature radius. $\emptyset^{0}$ is the view angle of the interface from the pipe centre, $\alpha$ is the angle between the vertical and the line connecting the interface at wall to the mid curvature point. Water height across the mid pipe is $h_{w}$ and the pipe diameter is denoted by $D$. Eqn (2) was obtained by simplifying the equation for interface curvature radius $(r)$ [3]

$$
r=c_{1} \times \exp \left[c_{2}-\left\{c_{3} \times\left(h_{w} / D\right)\right\}\right] .
$$

The constants $\mathrm{c}_{1}=0.0071, \mathrm{c}_{2}=5.16$ and $\mathrm{c}_{3}=3.555$ in the equation were calculated using the Eötvös $\left(\epsilon_{\mathrm{v}}\right)$ number as 37 and contact angle as $110^{\circ}$ which is specific to the current study. A geometrical relation was derived to relate the interface curvature radius (r) to the phase angle as shown in eqn (3) 


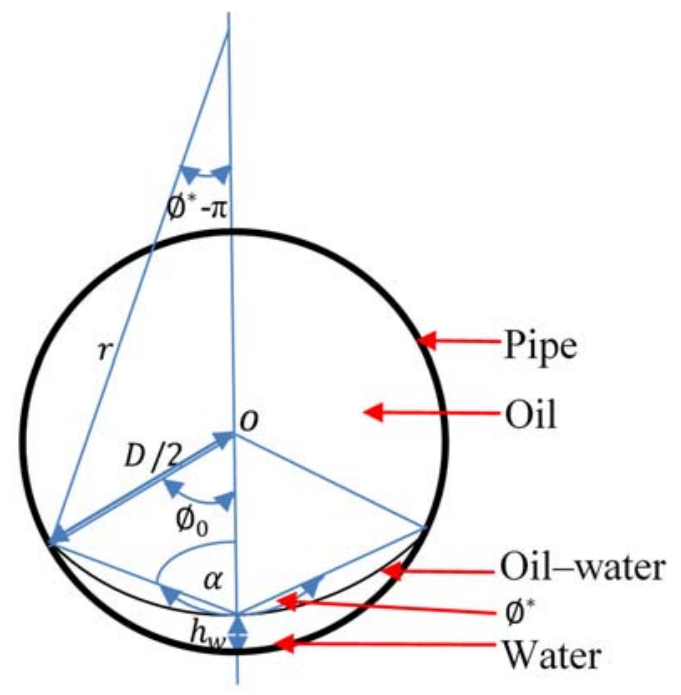

Figure 1: Geometrical description of the cross sectional view of oil-water stratified flow using constant curvature arc model.

$$
r=\frac{D / 2 \sin \omega}{\sin \left(\varnothing^{*}-\pi\right)}
$$

where $\emptyset^{*}=2(\pi-\alpha)$ and $\alpha=\tan ^{-1} \frac{D / 2 \sin \omega}{\left(D / 2^{-D / 2} \cos \omega-h_{w}\right)}$. Here, $\omega=\emptyset_{0}$ if the oil water interface at the pipe wall is below the half a pipe level and $\omega=\pi-\emptyset_{0}$, if the interface at the pipe wall is above. $\emptyset_{0}$ is calculated from the known interfacial margins at the pipe wall according to image analysis. Both the eqns (1) and (2) are functions of $h_{w}$ and plotting $r v s h_{w}$ yields the solution for the relevant $h_{w}$ which would enable to find the depth of the interfacial curvature.

\subsection{Numerical simulations}

A supplementary 3D CFD simulation was carried out using ANSYS Fluent 16.2 to model the interfacial shape of the stratified smooth (ST) oil-water flow in horizontal pipe $\left(U_{\text {mix }}=0.25\right.$ $\left.\mathrm{m} / \mathrm{s}, \lambda_{w}=0.5\right)$. The 3D meshed geometry and the boundary conditions are shown in Fig. 2. Phase inlet velocities were defined based on the mixture velocity and water cut. A short length of $0.5 \mathrm{~m}$ acrylic pipe was assumed to represent the transparent test section of the flow loop and to reduce the computational time. The physical properties of the test fluids were the same as mentioned in Section 2.1. A pressure-based, isothermal 3D simulation was performed under transient state for a sufficiently long period before extracting the results. The interfacial level at the pipe wall and the mid pipe were recorded at a plane defined $100 \mathrm{~mm}$ before the outlet plane. The multiphase, volume of fluid (VOF) model with implicit formulation was employed.

The standard k- $\varepsilon$ model with standard wall functions was considered for turbulence modelling and the curvature correction option was enabled. The continuum surface model (CSF) with wall adhesion option was enabled to model the phase interactions. An interfacial 


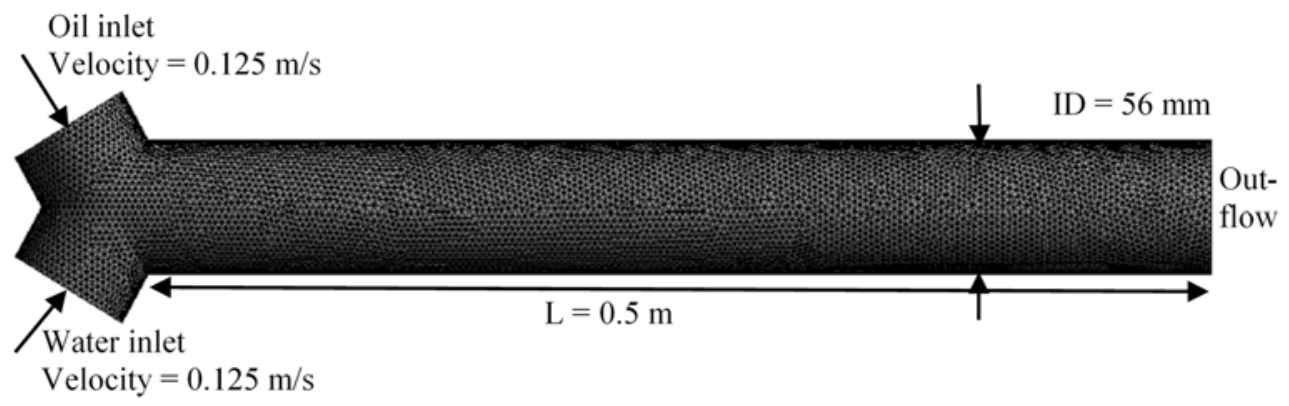

Figure 2: 3D geometry with boundary conditions.

tension value of $43 \mathrm{mN} / \mathrm{m}$ and a contact angle of $110^{\circ}$ for acrylic were considered, as for the experiments. The turbulent kinetic energy and its dissipation rate are calculated from a turbulence intensity of $5 \%$ and a hydraulic diameter of $56 \mathrm{~mm}$. The non-slip boundary condition was imposed on the wall of the pipe.

\section{RESULTS AND DISCUSSION}

\subsection{Observed interface for stratified smooth (ST) and stratified wavy (SW) flows}

Fig. 3 depicts the images with regarding to (ST) oil-water flow. Fig. 3(a) shows the crosssectional view of the time average tomogram obtained by the ECT system and Fig. 3(b) shows an instantaneous image from high-speed camera showing the longitudinal view of the oil-water flow along the pipe wall. Fig. 3(c) shows the time series of wave interface as read into Matlab, while Fig. 4 denotes the instantaneous volume fractions of water and oil across the cross section of the pipe according to the CFD simulations for ST flows. Fig. 5 illustrates the images with regarding to stratified wavy (SW) oil-water flow.

As illustrated in Fig. 3(a) and Fig. 5(a), the interface shown in time average ECT tomogram is not sharp and possess a considerable thickness. Even though, the camera images (see Fig. 3(b) and Fig. 5(b)) also show the interface with some thickness, this is negligible in comparison to what is given by ECT. Therefore, for high-speed images, the interface was obtained via detecting the brightest pixel relevant to each pixel column with time, with respect to a considered location. For ECT images, the interface was gained with an upper and lower limits via detecting the interfacial margins which vary between $100 \%$ water and $100 \%$ oil. The interface shown by the CFD cross sectional image is more definitive in comparison to ECT images and is defined via detecting the average volume fraction of water across the lines drown $1 \mathrm{~mm}$ away from the pipe wall and at the mid pipe.

\subsection{Interface level detection at the pipe wall by ECT, high speed image analysis and CFD}

Figs 6 and 7 show the interfacial level for the ST and SW flow at the pipe wall according to ECT and high-speed image processing techniques. The numerical simulations were performed only for the ST flow and the obtained results are plotted together with that of other techniques. The CFD results illustrated in Fig. 6 seems in very good agreement with that of high-speed image analysis. The data acquisition rate for high-speed camera images is $500 \mathrm{~Hz}$, while it is $100 \mathrm{~Hz}$ for ECT images and CFD. On the other hand, the pixel span is 


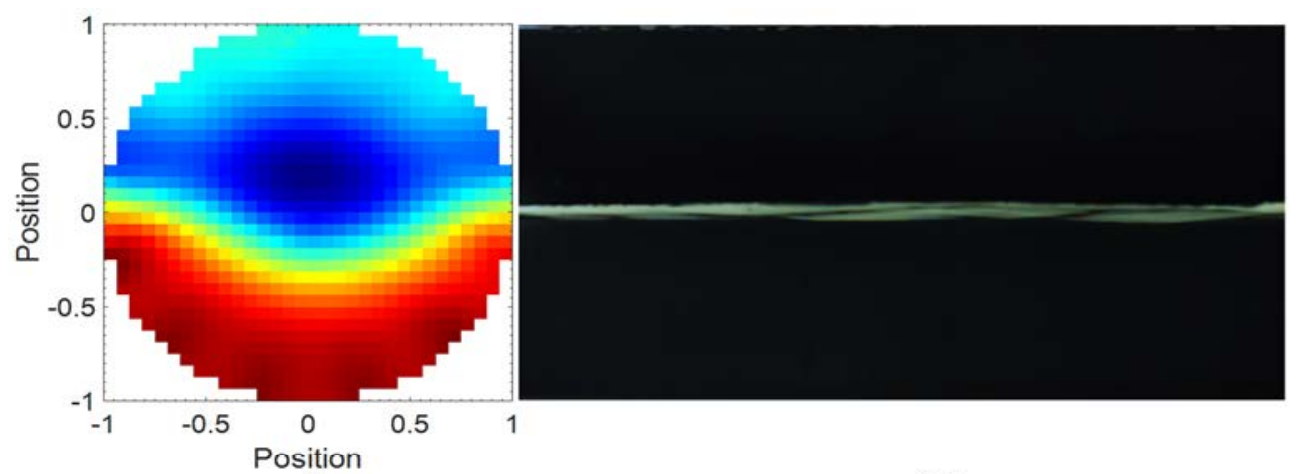

(a)

(b)

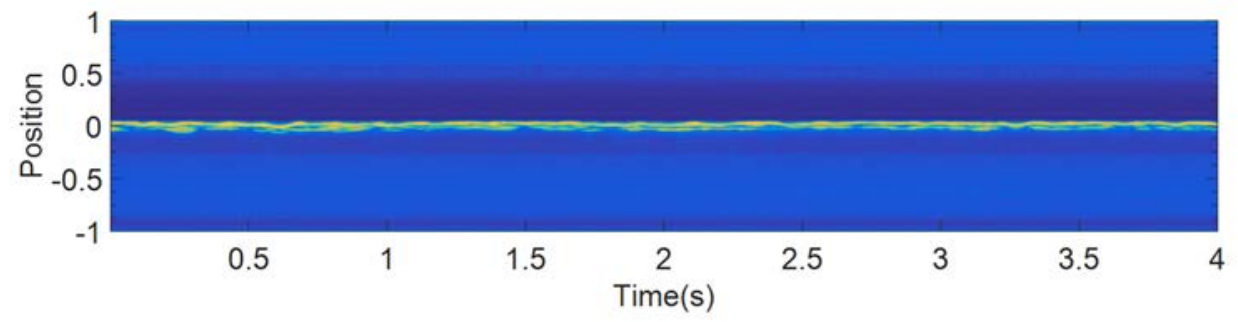

(c)

Figure 3: ST; $\theta=0^{\circ}, U_{m i x}=0.25 \mathrm{~m} / \mathrm{s}, \lambda_{w}=0.5$. (a) Cross sectional view of time average tomogram with the ECT system for oil (blue) and water (red); (b) Longitudinal view of still-camera image for the flow; (c) Oil-water level variation at the pipe wall varying with time given by analyzing the high-speed images.

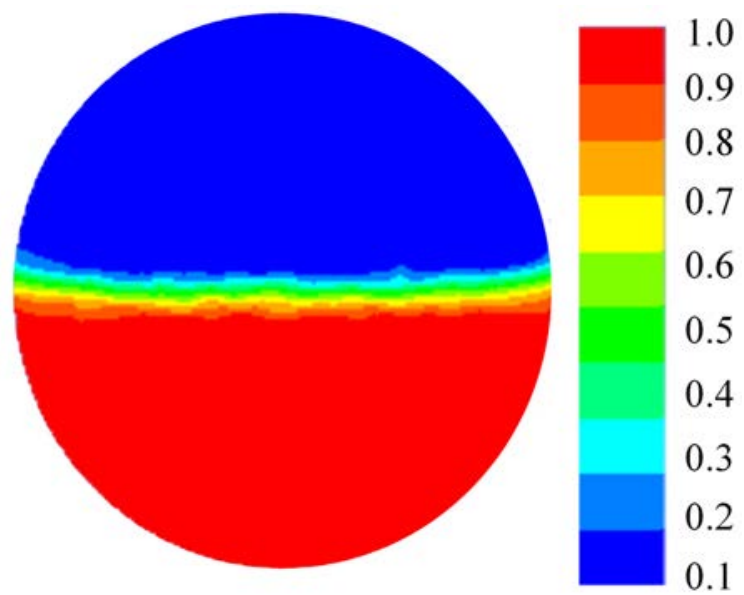

Figure 4: CFD prediction of the oil-water interface (ST; $\theta=0^{\circ}, U_{m i x}=0.25 \mathrm{~m} / \mathrm{s}, \lambda_{w}=0.5$ ). 


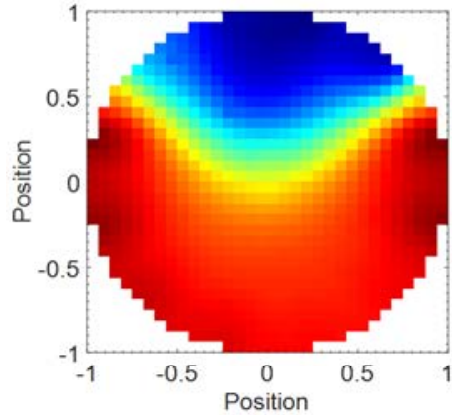

(a)

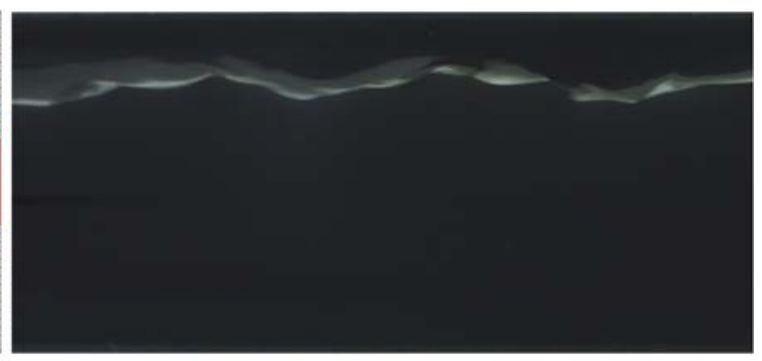

(b)

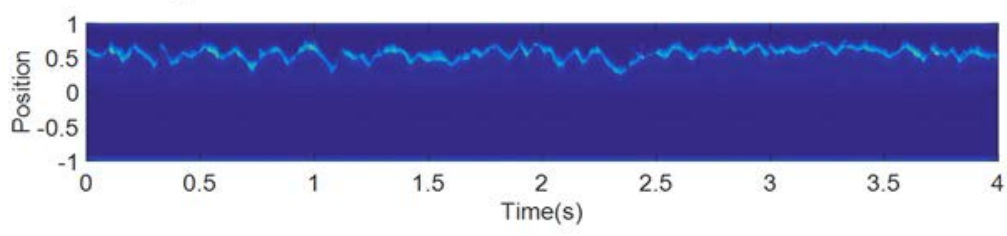

(c)

Figure 5: $\mathrm{SW} ; \theta=0^{\circ}, U_{m i x}=0.2 \mathrm{~m} / \mathrm{s}, \lambda_{w}=0.7$. (a) Cross sectional view of time average tomogram with the ECT system for oil (blue) and water (red); (b) Longitudinal view of the still-camera image for the flow; (c) Oil-water level variation at the pipe wall varying with time given by analyzing the high-speed images.

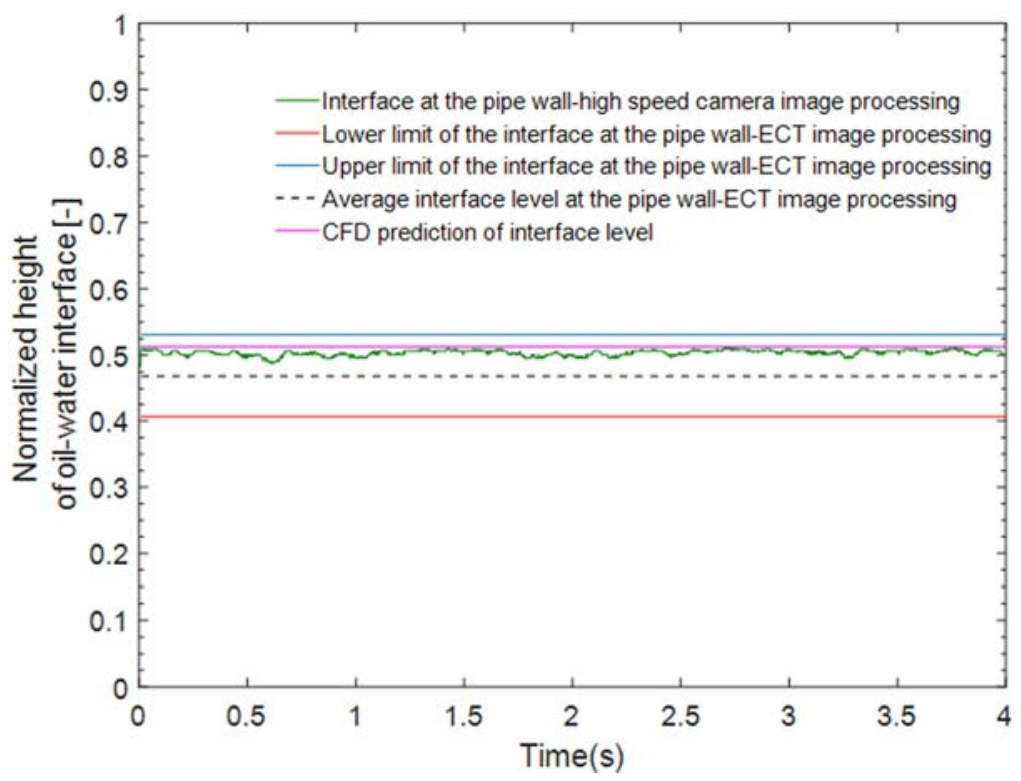

Figure 6: Oil-water interface level at pipe wall given by high-speed image processing and ECT image processing for ST flow at $\theta=0^{\circ}, U_{m i x}=0.25 \mathrm{~m} / \mathrm{s}, \lambda_{w}=0.5$. *Data acquisition frequency for ECT is $100 \mathrm{~Hz}$ and for high-speed imaging is $500 \mathrm{~Hz}$. 


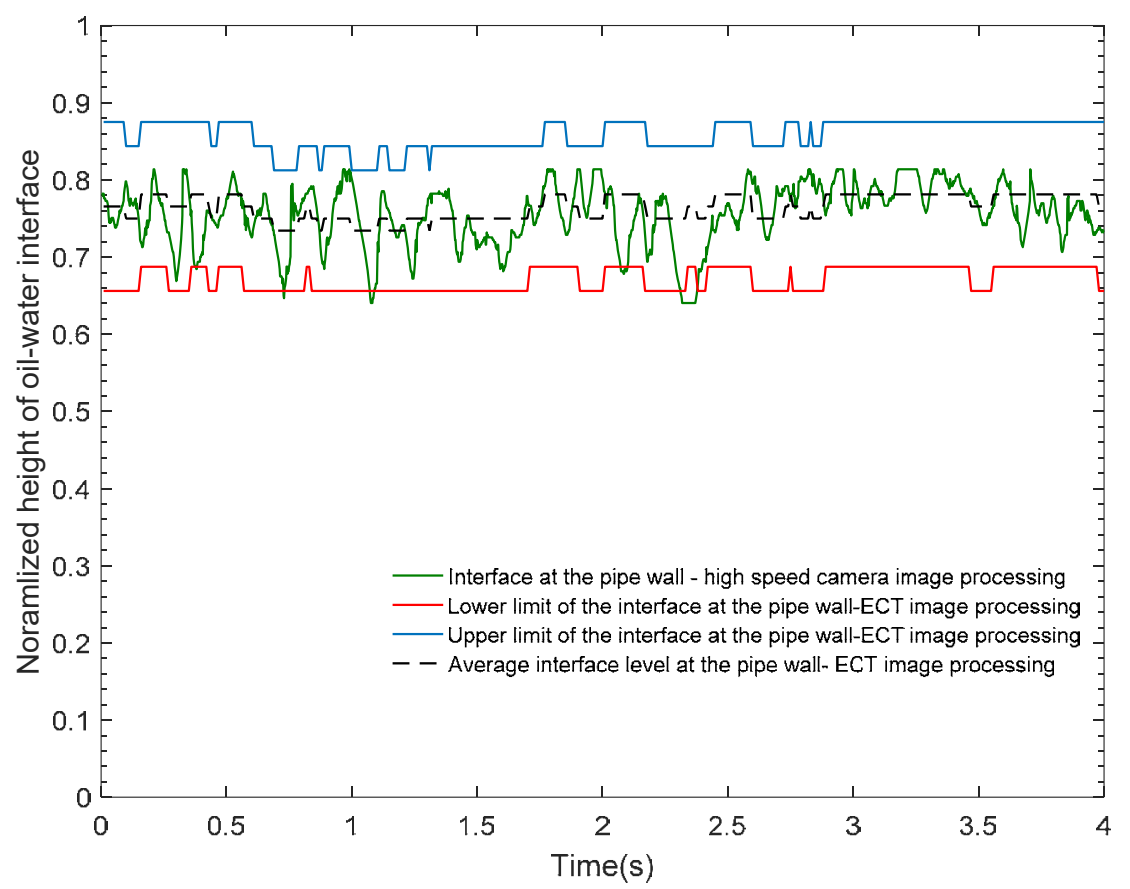

Figure 7: Oil-water interface level at pipe wall by high-speed image processing and ECT image processing for SW flow at $\theta=5^{\circ}, U_{m i x}=0.2 \mathrm{~m} / \mathrm{s}, \lambda_{w}=0.7$.

$1024 \times 352$ for high-speed camera images and it is $32 \times 32$ for ECT images. The CFD geometry was meshed with an element size of $3 \mathrm{~mm}$ and the total number of cells were 543,962 . Therefore, ECT data seem quite coarse in comparison to the high-speed camera images and CFD images along both the x-direction (time axis) and y-direction (normalized interfacial height). The longitudinal interfacial level fluctuations are negligible for ST flow while the periodic level fluctuations inherited to SW flow is noticeable in data captured by both imaging techniques. In addition, a few drops of water present in oil phase can make significant change in the measured capacitance values that directly affect the ECT images.

According to Figs 6 and 7, the interfacial level at the pipe wall, obtained by camera image analysis and CFD stay within the interfacial margins given by time average ECT tomogram. For ST flow, ECT-IA (0.47) under-predicts the average interfacial level predicted by H-IA $(0.5)$ only by $6 \%$. Interfacial level predicted by CFD $(0.51)$ is in good agreement with that of H-IA.

For SW flow, the calculated average interfacial level predicted by H-IA (0.76) is equivalent to the mean value of average interfacial level predicted by ECT-IA (0.76). Therefore, ECT can predict the average interfacial oil-water level at the pipe wall with higher accuracy.

\subsection{Interface level detection at the mid-pipe}

The interfacial level at the mid pipe was analytically found by solving eqn (2) and (3). Figs 8 and 9 show the interface level at the mid pipe according to ECT image processing for ST and SW flow respectively. 


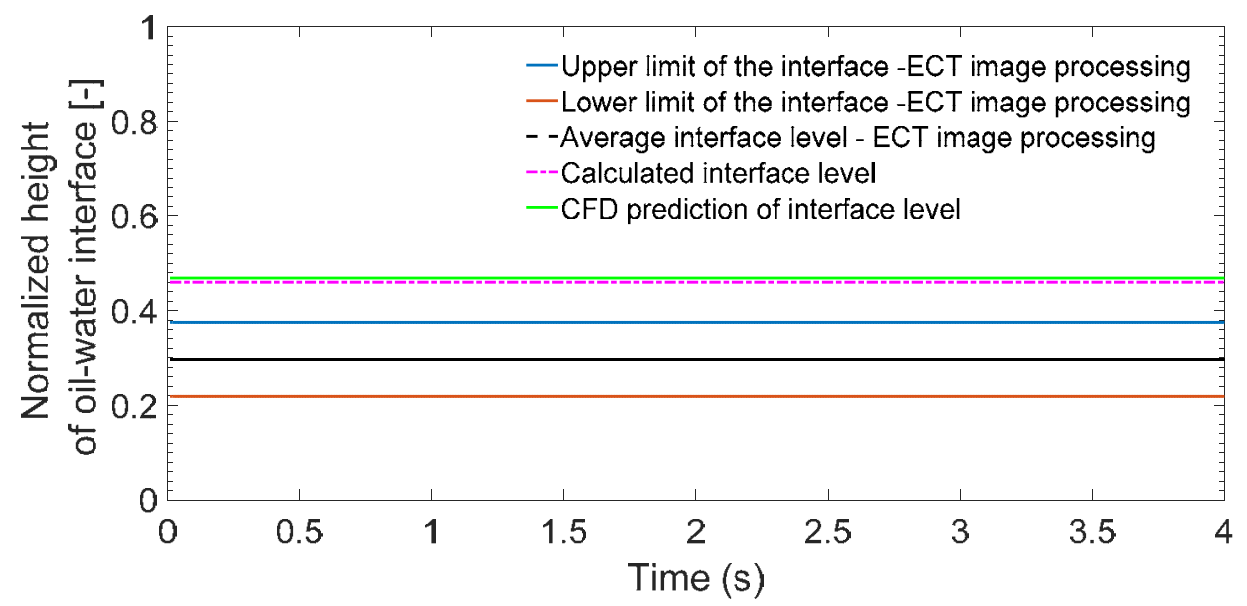

Figure 8: Oil-water interface level at mid-pipe given by ECT image processing and solving curvature radius equations for ST flow at $\theta=0^{\circ}, U_{m i x}=0.25 \mathrm{~m} / \mathrm{s}, \lambda_{w}=0.5$.

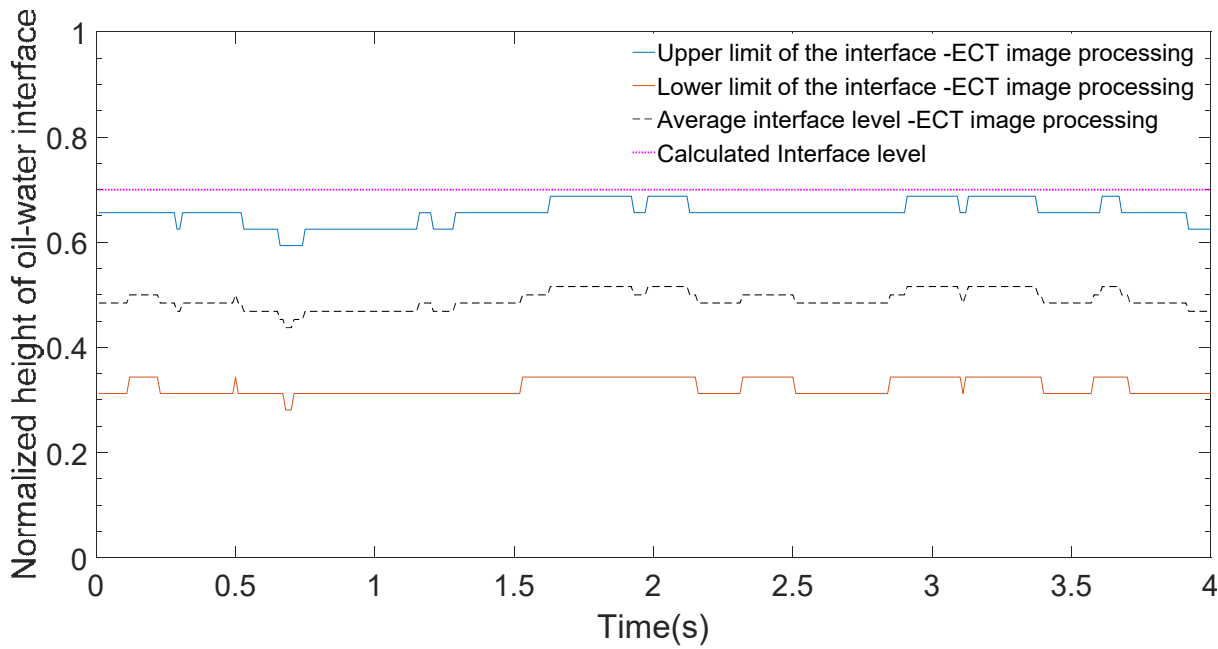

Figure 9: Oil-water interface level at mid-pipe by ECT image processing and solving curvature radius equations for $\mathrm{SW}$ flow at $\theta=5^{\circ}, U_{m i x}=0.2 \mathrm{~m} / \mathrm{s}, \lambda_{w}=0.7$.

Additionally, Fig. 8 contains the results from CFD simulations for the ST flow. The calculated interfacial level is plotted as a reference line together with the ECT data, for comparison. According to ECT, the mean value of average oil-water interfacial height at mid pipe is 0.3 for ST flow and 0.49 for SW flow. According to the calculated data, this is 0.46 for ST flow and 0.7 for SW flow when considered in normalized scale. For ST flow, the interfacial level at mid pipe predicted by CFD (0.47) is in well agreement with that of the calculated value (0.46) using constant curvature arc model. Therefore, it can be stated that, ECT under-predicts the calculated interfacial height by $35 \%$ for ST flow and $30 \%$ for SW flow. 


\subsection{Interfacial curvature}

Table 1 shows the summary of the results given by each technique used for interfacial oilwater level detection at pipe wall and the mid pipe.

As can be noted, the interfacial margins at the pipe wall can be accurately predicted by ECT, while it fails at the mid pipe region. The calculations and CFD simulations confirm the existence of an interfacial curvature. However, the curvature depth at mid pipe is considerably over-predicted by ECT results for both the ST and SW flow cases. Assuming that the most accurate interfacial level at the pipe wall is predicted by $\mathrm{HIA}_{\mathrm{w}}$ and at the mid pipe by $\mathrm{Cal}_{\mathrm{m}}$, ECT over-predicts the interfacial curvature depth by 4.3 times for both the flow cases. These findings are well agreed with the numerical simulations performed by CFD.

\section{CONCLUSION}

Even though it is traditionally accepted that the interfacial curvature is seen only in capillary tubes, it can exist in gravity-reduced systems where the surface forces are significant such as liquid-liquid flows of matching densities.

Oil-water flows with density ratio of 0.8 and viscosity ratio of 1.6 were studied for interfacial shape, using ECT, high-speed camera imaging, model calculations and by CFD.

High-speed camera images were analysed to compare the interfacial level at pipe wall given by ECT and the results showed very good agreement for both the ST and SW flow cases.

Table 1: Summary of the results.

\begin{tabular}{|c|c|c|c|}
\hline \multicolumn{2}{|c|}{ Interfacial level data } & ST & SW \\
\hline \multirow{3}{*}{$\begin{array}{l}\text { Interfacial water height at pipe } \\
\text { wall (mm) }\end{array}$} & $\mathrm{ECT}_{\mathrm{w}}$ & 26.3 & 42.6 \\
\hline & $\mathrm{HIA}_{\mathrm{w}}$ & 28 & 42.6 \\
\hline & $\mathrm{CFD}_{\mathrm{w}}$ & 28.7 & - \\
\hline Phase angle- $\emptyset_{0}\left({ }^{\circ}\right)$ & View angle of the interface & 90 & 120 \\
\hline \multirow{3}{*}{$\begin{array}{l}\text { Interfacial water height at } \\
\text { mid-pipe (mm) }\end{array}$} & $\mathrm{ECT}_{\mathrm{m}}$ & 16.8 & 27 \\
\hline & $\begin{array}{l}\mathrm{Cal}_{\mathrm{m}}(\text { Solving for curvature } \\
\operatorname{radius}(\mathrm{r}))\end{array}$ & 25.8 & 39 \\
\hline & $\mathrm{CFD}_{\mathrm{m}}$ & 26.3 & - \\
\hline \multirow{5}{*}{$\begin{array}{l}\text { Depth of the interfacial } \\
\text { curvature }(\mathrm{mm})\end{array}$} & $\mathrm{ECT}_{\mathrm{w}}-\mathrm{ECT}_{\mathrm{m}}$ & 9.5 & 15.6 \\
\hline & $\mathrm{HIA}_{\mathrm{w}}-\mathrm{ECT}_{\mathrm{m}}$ & 11.2 & 15.6 \\
\hline & $\mathrm{ECT}_{\mathrm{w}}-\mathrm{Cal}_{\mathrm{m}}$ & 0.5 & 3.6 \\
\hline & $\mathrm{HIA}_{\mathrm{w}}-\mathrm{Cal}_{\mathrm{m}}$ & 2.2 & 3.6 \\
\hline & $\mathrm{CFD}_{\mathrm{w}}-\mathrm{CFD}_{\mathrm{m}}$ & 2.4 & - \\
\hline
\end{tabular}

*Here, HIA-High speed camera image analysis, w-value at the wall, m-value at the mid pipe, Cal-calculated value by solving the equations for curvature radius, $\mathrm{CFD}$ - interfacial level obtained by numerical simulations. 
Having figured the interfacial level at the pipe walls, the depth of the interfacial curvature at the mid pipe was calculated by solving the simultaneous equations for curvature radius (r) using the constant curvature arc model.

The calculated interfacial level at the mid pipe predicts much lesser depth of interfacial curvature at the mid pipe in comparison to that of ECT predictions. The depth of the curvature radius predicted by ECT is 4.3 times that of the calculated value for both the ST and SW flows.

Finally, the interfacial curvature was investigated for ST flow, using CFD and the results are in very good agreement with the calculated results using constant curvature arc model by confirming the existence of a slight interfacial curvature.

Therefore, it can be concluded that the average interfacial level calculated using ECT, can predict the interfacial level at pipe wall with a higher accuracy while it can over-estimate the depth of interfacial curvature at mid-pipe. Deflection of the electric flux lines when they move through media of high permittivity contrast, like oil and water, could be the possible reason for this observed scenario. Especially the density of electric flux lines are higher at wall areas in comparison to the mid pipe area and hence the mid pipe area could be more susceptible to the distortions.

\section{ACKNOWLEDGEMENTS}

The authors gratefully acknowledge Professor Morten C. Melaaen, Dean Faculty of Engineering, Vice Dean Randi T. Holta, Associate Professor Amaranath Sena Kumara and Professor Saba Mylvaganam of USN (University of South-Eastern Norway) for providing the opportunity and the test facilities to perform this research. We appreciate the help provided by Docent Finn Aakre Haugen in providing us with the data logging and control programs and updating them in conjunction with the measurements done in the multi-flow testing facilities of USN. Sincere thanks are extended to Dr Chaminda Pradeep and laboratory engineer, Fredrik Hansen for their support in assisting with the experiments, sharing knowledge and Dr Andre. V. Gaathaug for providing the high-speed camera facilities.

We are grateful to the Government of Norway (Kunnskapsdepartementet (KD)) and the 'Statoil Akademia-UiS' cooperation project for funding and providing support to this research.

\section{REFERENCES}

[1] Taitel, Y. \& Dukler, A.E., A model for predicting flow regime transitions in horizontal and near horizontal gas-liquid flow. American Institute of Chemical Engineers, 22(1), pp. 47-55, 1976.

[2] Brauner, N., Moalem Maron, D. \& Rovinsky, J., A two-fluid model for stratified flows with curved interfaces. International Journal of Multiphase Flow, 24(6), pp. 975-1004, 1998.

[3] Rodriguez, O.M.H. \& Baldani, L.S., Prediction of pressure gradient and holdup in wavy stratified liquid-liquid inclined pipe flow. Journal of Petroleum Science and Engineering, 96-97, pp. 140-151, 2012.

[4] Sunder Raj, T., Chakrabarti, D.P. \& Das, G., Liquid-liquid stratified flow through horizontal conduits. Chemical Engineering \& Technology, 28(8), pp. 899-907, 2005.

[5] Brauner, N., Rovinsky, J. \& Moalem Maron, D., Determination of the interface curvature in stratified two-phase systems by energy considerations. International Journal of Multiphase Flow, 22(6), pp. 1167-1185, 1996.

[6] Gorelik, D. \& Brauner, N., The interface configuration in two-phase stratified pipe flows. International Journal of Multiphase Flow, 25(6), pp. 977-1007, 1999. 
[7] Ng, T.S., Lawrence, C.J. \& Hewitt, G.F., Interface shapes for two-phase laminar stratified flow in a circular pipe. International Journal of Multiphase Flow, 27(7), pp. 1301-1311, 2001.

[8] Ng, T.S., Lawrence, C.J. \& Hewitt, G.F., Laminar stratified pipe flow. International Journal of Multiphase Flow, 28(6), pp. 963-996, 2002.

[9] Perera, K., Pradeep, C., Mylvaganam, S. \& Time, R.W., Imaging of oil-water flow patterns by Electrical Capacitance Tomography. Flow Measurement and Instrumentation, 56, pp. 23-34, 2017. 